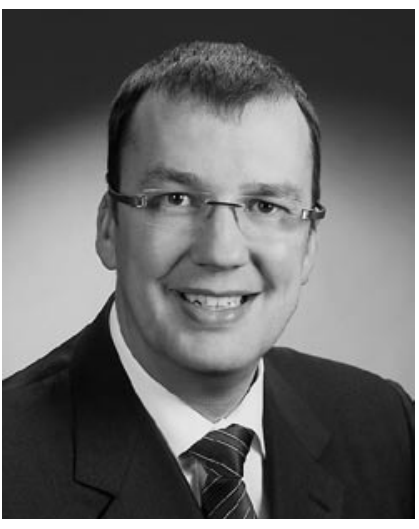

Utz Schäffer

\section{Der Rollenwandel des CFO}

\author{
Liebe Leser,
}

über Controller wird viel geschrieben, auch in der ZfCM. Über "Chief Financial Officers" und Finanzvorstände finden sich hingegen vergleichsweise wenige Publikationen. Ein ähnliches Bild ergibt sich für die universitäre Lehre: Über Controller wird viel gesprochen, über CFOs, das Zusammenspiel der verschiedenen Funktionen des Finanzbereichs und die Unternehmenssteuerung hingegen weniger. Dabei scheint die Bedeutung der CFOs in den letzten Jahren gestiegen zu sein. Ihre Rolle und ihr Selbstverständnis haben sich in den meisten Unternehmen sichtbar geändert. Neue Aufgaben sind hinzugekommen, die strategische Weiterentwicklung des Unternehmens wurde in vielen Fällen zu einer Kernaufgabe. Als Counterpart des CEOs haben heute viele CFOs die Fäden (mit) in der Hand.

Über die Gründe, warum so wenig über CFOs geschrieben wird, lässt sich nur spekulieren. Vielleicht sind die Zugangsbarrieren für viele Forscher prohibitiv hoch: welcher CFO füllt schon einen längeren Fragebogen (selbst) aus? Vielleicht liegt es auch daran, dass an Hochschulen kein Lehrstuhlinhaber von Haus aus "zuständig“ ist und fachübergreifende Zusammenarbeit eher die Ausnahme darstellt: der Aufgabenbereich des CFO umfasst eben mehr als nur Controlling, nur Accounting oder nur Finance.

Wie auch immer - es ist an der Zeit, dass wir uns mit dem vorliegenden Schwerpunktheft dem Rollenwandel des CFO widmen. Der einleitende Beitrag eines Forscherteams vom Institut für Management und Controlling der WHU untersucht Karrierewege von CFOs in Dax-30-Unternehmen. Dabei werden von den Autoren die Karriereverläufe der CFOs von 1998 mit den Laufbahnen der CFOs von 2008 verglichen: Wussten Sie zum Beispiel, dass mit Rainer Feuerhake von der TUI lediglich eine Person sowohl 1998 als auch 2008 CFO eines Dax-30-Unternehmens war? Im Anschluss schreibt mit Jürgen Daum ein Autor über die Entwicklung der CFO-Rolle in europäischen Unternehmen, der sich schon lange Jahre mit dem Aufgabenfeld des CFO und der Neuausrichtung der Finanzorganisation befasst. Er argumentiert, dass das neue Aufgabenprofil des CFO ohne eine fundamentale Transformation des Finance\&Control-Bereichs nicht zu leisten ist. Damit hat das veränderte Rollenbild des CFO nicht zuletzt auch signifikante Auswirkungen auf das Berufsbild des Controllers. Mit Christian Göseke und Ekkehard zur Mühlen bringen schließlich zwei CFOs selbst ihre Perspektive ein. Der Beitrag von Christian Göseke befasst sich im Schwerpunkt mit den Erfolgsfaktoren für die Zusammenarbeit von CEO und CFO, Ekkehard zur Mühlen diskutiert im Interview mit der Redaktion die Rolle des Controllers im Finanzbereich.

Aber auch über den Rollenwandel des CFO hinaus liefert der vorliegende Band spannende Beiträge zu internen Kontrollsystemen in mittelgroßen Unternehmen, zur professionellen Gestaltung des Beteiligungscontrollings sowie zum Performance Board als Werkzeug der Strategieumsetzung in einem internationalen Fahrzeugbaukonzern. Viel Spaß bei der Lektüre wünscht Ihnen

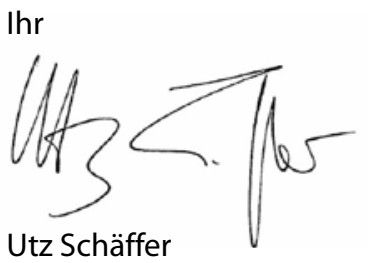

Utz Schäffer 